\title{
La productividad territorial y el rol de la cartografía en la defensa del parque Reforma Social, Ciudad de México
}

\section{Territorial productivity and the role of cartography in the defense of the Social Reform Park, Mexico City}

\author{
Silvia Carbone \\ ORCID: https://orcid.org/0000-0001-8388-120X \\ Correo electrónico: kechivis@gmail.com
}

\section{Stephane Couturier \\ ORCID: https://orcid.org/0000-0003-0564-879X}

Filiación: Instituto de Geografía, Universidad Nacional Autónoma de México, Ciudad de México, México.

\section{Resumen}

El impulso del modelo económico neoliberal en la Ciudad de México ha estimulado la inversión de capital en obras e intervenciones, las cuales desencadenan problemas entre los actores sociales involucrados. El siguiente artículo propone un análisis del conflicto en torno al carácter público del parque Reforma Social, ubicado en la alcaldía Miguel Hidalgo, en el que asistimos al enfrentamiento de intereses entre los vecinos -movilizados para conservar el parque-y un grupo de actores que tiene planeado desarrollar un proyecto inmobiliario. En el marco de las pugnas territoriales que atraviesan esta metrópolis, analizamos la controversia bajo el supuesto de que esta modifica las relaciones de poder previas al estallido de la confrontación y tiene una productividad. Después de delimitar el conflicto como situación, se recupera la voz de los actores, sus argumentos y posicionamientos, desde una lectura que analiza las alianzas, así como la capacidad de los vecinos de proponer nuevas estrategias de defensa territorial. Dentro de los resultados, se evidencia cómo al mismo tiempo que se incrementa la cohesión de los residentes, se reconoce el papel protagónico desempeñado por la cartografía, en particular, en lo que concierne al proceso de apropiación y de proyección territorial experimentado por los vecinos de la colonia

\section{Palabras clave}

Espacio público, pandemia de COVID-19, urbanizaciones informales, vulnerabilidad

\begin{abstract}
The promotion of the neo-liberal economic model in Mexico City has stimulated capital investment, as works and interventions, which trigger conflicts between the social actors involved. This article proposes an analysis of the situation surrounding the public nature of the Reforma Social Park, located in the Miguel Hidalgo Mayor's Office. We show the confrontation of interests between the neighbors, which are mobilized to conserve the park and a group of business actors who plans to develop a real estate project. In the framework of the territorial struggles that cross the metropolis, we analyze the conflicts under the assumption that they modify the power relations before the outbreak of the confrontation. After delimiting the conflict as a situation, we will introduce the voice of the actors, their arguments and positions. Also, we will propose a dynamic reading that analyzes alliances, and the neighbors' capacity to offer new territorial defense strategies. Throughout the analysis, we see how, at the same time as the cohesion of the neighbors increased, the leading role that cartography has played is recognized, particularly about the process of appropriation and territorial projection, which the inhabitants experience.
\end{abstract}

\section{Keywords}

Informal urbanizations, COVID-19 pandemic, public space, vulnerability 


\section{Introducción}

Con el impulso del modelo económico neoliberal, los gobiernos de las metrópolis del Sur Global han ido dejando su papel como garantes del bien común, para asumir el rol de gestor de los procesos urbanos bajo un modelo de desarrollo orientado por la lógica empresarial (Bredenoord, Lindert \& Smets, 2014; Gwinne, 1999). $\mathrm{El}$ alza del valor del suelo en los corredores centrales de la Ciudad de México ha estimulado la inversión de capital, desencadenando conflictos entre el sector empresarial y los vecinos que ven amenazado su estilo de vida y la calidad de su entorno (GeoComunes, 2020; Morales Guzmán, 2017). Estas tensiones evidencian las visiones encontradas acerca de los criterios subyacentes al devenir urbano y a las normas que lo regulan: los procedimientos para cambio de uso de suelo, la toma de decisiones, la negociación de intereses encontrados y los alcances de la gobernanza.

En el siguiente artículo se analiza el conflicto en torno al carácter público del parque ubicado en la colonia Reforma Social, en la alcaldía Miguel Hidalgo de la Ciudad de México (Figura 1) ${ }^{1}$. Una controversia que enfrentó a los vecinos de la colonia, movilizados en defensa del parque, con un grupo de actores que buscaba desarrollar un proyecto inmobiliario. El conflicto gira en torno a un procedimiento legal de amparo que pone en tela de juicio el carácter público del predio.

El parque es, por lo general, un espacio jurídicamente público que conjuga la dimensión ambiental con la recreativa; pero, en nuestro caso, articula estas cualidades con la sociopolítica al constituir el pretexto que congrega a los vecinos para la acción. Por ello, más que enfocarnos en los procesos de privatización resultantes de prácticas excluyentes o en la implementación de modelos de gestión mercantil, indagaremos en los valores subyacentes a la controversia que animan a los actores sociales, analizando la relación entre los vecinos y las instituciones gubernamentales y valorando la eficacia de las estrategias implementadas.

El horizonte de análisis es contemporáneo: el conflicto estalló en el año 2010, cuando el delegado de la alcaldía Miguel Hidalgo ${ }^{2}$ anunció la ejecución de la sentencia que reconocía la propiedad privada del predio. En esa coyuntura, los vecinos irrumpieron en la esfera pública con expresiones de protesta (Azuela, 2014; Melé, 2003, 2016), las cuales se analizarán como momentos de incidencia colectiva en el marco de un sistema de acción. Este es un acercamiento que ayuda a reconocer los valores que animan los agentes, sin descartar eventuales implicaciones estructurales (Melé, 2003; Melucci, 1999; Simmel, 2010).

El caso se expuso en periódicos de alcance urbano, en el ámbito académico y fue motivo de una ponencia acerca de la utilidad de los Sistemas de Información Geográfica (SIG), desde la perspectiva de los movimientos sociales urbanos $^{3}$. Nuestra propuesta, en cambio, analiza la controversia en el marco de los conflictos territoriales que atraviesan Ciudad de México, enfocándonos sus efectos. La disputa modifica las relaciones de poder, poniendo en cuestión el orden previo al estallido (Simmel, 2010). Nuestra hipótesis plantea que el conflicto por el parque implica, entre otras cuestiones, una productividad territorial; además, en este proceso el tema cartográfico desempeña un papel estratégico. Nuestras preguntas de investigación son: ¿Qué está en juego con la privatización del parque Reforma Social (RS)? ¿Qué efectos de orden

1 Reforma Social está localizada en la alcaldía Miguel Hidalgo, al sur del Campo Militar No. 1; colinda con las colonias Lomas de Chapultepec, Lomas de Barrilaco y Polanco hacia el sur; al oriente con el municipio de Naucalpan. Es catalogada como colonia popular por el Programa Delegacional de Desarrollo Urbano (PDU-MH), de acuerdo con el Censo 2010 de INEGI, cuenta con una población de 5.195 habitantes.

2 Demetrio Sodi gobernó como delegado de la Miguel Hidalgo (2009-2012) por el Partido de Alianza Nacional (PAN). Le sucedió Víctor Hugo Romo (Partido Revolución Democrática-PRD) y, en 2015, Xochitl Gávez (PAN). En 2018, Romo (Movimiento de Regeneración Nacional, MORENA) volvió a ser electo alcalde. A su vez, Marcelo Ebrard Casaubón (PRD) gobernó la Ciudad de México desde 2006 hasta 2012. Le siguió Miguel Ángel Mancera (PRD) hasta el año 2018, cuando le sucedió Claudia Sheinbaum Pardo (MORENA).

3 Sobre este tema ver: la presentación de Piceno, Couturier y Ricárdez (2014); la tesis de Piceno (2013), que trata el conflicto desde la perspectiva de los movimientos sociales; y la tesis de Navarro Jiménez (2013), que proporciona una reconstrucción del proceso de producción de la colonia. 
Figura 1

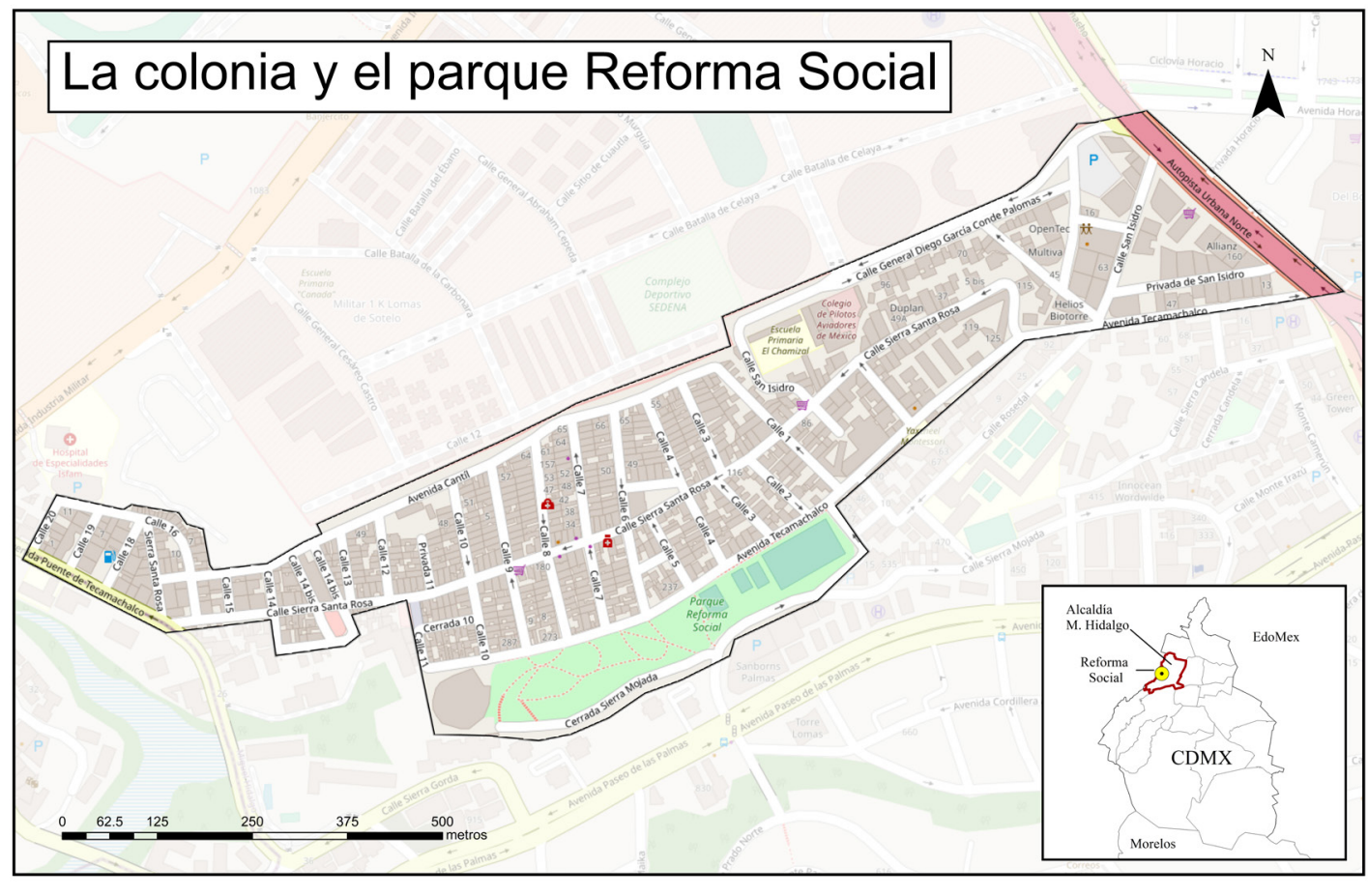

Nota. Gentileza de Gabriel Eguiluz (basado en OpenStreetMap).

territorial produjo el conflicto? ¿Cuál es el papel de la cartografía en la defensa del parque?

El artículo se estructura en tres partes: la primera aborda la introducción con los planteamientos y la estrategia metodológica, así como los conceptos utilizados: el conflicto como situación, su traducción territorial, el proceso de popularización de la herramienta cartográfica. Después se analiza el caso del parque recuperando la voz de los agentes y posicionamientos desde una lectura dinámica (Azuela, 2014; Merlinsky, 2013; Morales Guzmán, 2016); para luego concluir con algunas reflexiones acerca de los efectos del conflicto en torno al parque RS.

\section{El territorio en disputa y los efectos del conflicto. Delimitaciones conceptuales}

Nos interesan las movilizaciones con una base territorial en la Ciudad de México, donde los movimientos sociales urbanos han sido protagonistas. Las principales reflexiones - de corte marxista - orientan el análisis de estos movimientos a su capacidad para incidir en los procesos urbanos (Melé, 2016). Es la perspectiva desarrollada por Castells (1974), enriquecida luego desde la geografía crítica por autores como Harvey (2013), quien posiciona los movimientos en el centro de los procesos de impacto urbano, haciendo hincapié en la dimensión económica. Otras vetas enfocan las contradicciones que interesan a las grandes ciudades arrojando luz al análisis del vínculo entre el Estado y los sectores sociales, en el marco de un proceso de cambio estructural (Melé, 2016, p. 4). Esta perspectiva es criticada por concebir al Estado como entidad homogénea y no como un campo de disputa entre grupos; además, por adoptar un modelo analítico inscrito en un momento histórico en que el Estado ocupaba otro lugar en las relaciones entre grupos societarios (Melé, 2016).

Recientemente, esta mirada se enriquece con nuevas proposiciones: para la capital mexicana, Zamorano reflexiona sobre los "grupos de acción colectiva interesados, no en tomar el poder del Estado, sino en construir un mundo diferente desde los márgenes" (Zamorano, 2019, p. 22) y propone lo prefigurativo como herramienta para contemplar un espectro amplio de agrupaciones: identifica agrupaciones a-partidistas - los movimientos sociales emergentes- cuya composición interclasista promueve relaciones horizontales entre sus miembros. Ello enriquece esta perspectiva que, aunque capta las convergencias de las disputas desde un marco unificador, 
descuida otras modalidades de organización y acción (Zamorano, 2019). Consecuentemente, proponemos contemplar una variedad de agrupaciones de distintos sectores socioeconómicos, las que están activas en diversos territorios (Morales Guzmán, 2017), si los sectores populares demandan el mejoramiento del hábitat, estos grupos reaccionan a una amenaza. Además, observamos un proceso de apropiación de las herramientas digitales por parte de la ciudadanía, entre las cuales la cartografía gana protagonismo.

Este encuadre, planteado por autores como Melé (2008, 2016) y Azuela (2014) entre otros, y aplicado en distintos contextos geográficos, resalta diversos efectos del conflicto: la hibridación de las relaciones entre agentes sociales y gubernamentales, la productividad territorial, jurídica y política (Melé, 2016) o los procesos de territorialización, de construcción del ámbito público y actualización del derecho (Azuela, 2014).

\section{Articulando sociología del conflicto y territorio}

El conflicto expresa una tensión entre unidad y alejamiento que - mientras no crezca demasiado- es productiva en el sentido de la socialización (Simmel, 2010). Bajo esta premisa realizaremos un avance, valorando las alianzas o contraposiciones existentes entre los grupos, así como en su interior. Para articular esta noción sociológica con la lectura geográfica, recuperamos la geografía social francesa que se ha interesado en los conflictos desde un ángulo territorial (Aliste, 2010; Di Méo, 1998; Di Méo y Buléon, 2005). De la noción de territorio, que visibiliza las relaciones de poder entre los grupos, destacamos su cualidad multidimensional desde tres factores: el contexto geográfico; la dimensión individual de inserción en el entorno geográfico; y su carácter social, que implica valores y representaciones colectivas (Di Meo, 1998). Ello resalta la inteligibilidad de la red de territorios tejida por los sujetos desde la experiencia del espacio vivido, evidenciando la articulación entre pasado y futuro. Por su parte la territorialidad refiere a estrategias y prácticas que los agentes implementan: es la expresión geográfica del ejercicio del poder (Raffestin, 1999, citado en De Matteis y Governa, 2005), resultado de prácticas y conocimientos, de las relaciones que cada agente mantiene con los otros y con su territorio (De Matteis y Governa; 2005, p. 44).

En nuestro caso asistimos a la disputa de territorialidades, donde la ciudadanía, mediante el despliegue de argumentos y grados de apropiación, se enfrenta a la presión empresarial que busca orientar el devenir urbano. Entre las estrategias utilizadas destaca el uso de las TIC (Tecnologías de Información y Comunicación), cuyo protagonismo corre en paralelo con la apropiación de estrategias comunicativas y el uso de las redes sociales. La digitalización de la información, la aparición de aplicaciones y de programas interactivos incide en la comunicación entre instituciones y ciudadanía, fortaleciendo la exigencia de políticas de transparencia y control ciudadano.

\section{Cartografía social, mapeo participativo y ejercicio ciudadano}

Los mapas están cada vez más presentes en nuestras vidas: ya sea que se trate del sistema de trenes del metro o de las aplicaciones utilizadas para fines de movilidad. Gracias a la difusión de la red y, particularmente de la web2, (Osorno Covarrubias, Couturier y Ricárdez, 2015) son ya parte de nuestra cotidianidad. Además, la creciente capacidad para cruzar importantes cantidades de datos, y la difusión de aplicaciones que permiten a los usuarios crear los contenidos, fomenta la popularización de la cartografía que, después de estar históricamente ligada al ejercicio del poder, hoy por hoy se vuelve un instrumento en mano de los habitantes (Delgado Peña, Campoy Gómez y Subires Mancera, 2015).

Desde la modernidad, el mapa es la representación del territorio según reglas científicas de proyección, orientación, escala y convenciones simbólicas (Connolly, 2009, pp. 58-59). Sin embargo, una delimitación más amplia, propone el mapa como "cualquier representación gráfica que facilita la comprensión espacial de cosas, conceptos, condiciones, procesos o eventos en el mundo humano" (Harley, 2005, p. 16) y que pone en contacto al hombre con su entorno. La consciencia espacial incide 
en los procesos sociales, enfatizando que los mapas y el lenguaje construyen nuestra percepción del territorio (Connolly, 2009, p. 62) y que la territorialidad, además de la apropiación, corresponde a un proceso de identificación y pertenencia (Mora-Paez, p. 11).

El mapa distorsiona la realidad para aprenderla y comunicarla (Dos Santos, 1991), por ello es selectivo, simbólico; su valoración implica el contexto social, histórico y técnico y su autoría resulta importante, así como su accesibilidad y difusión (Connolly, 2009). Situado en un proceso comunicativo, el mapa conlleva una ideología (Barragán-León, 2017): el cuestionamiento de la relación entre discurso, conocimiento y poder y, consecuentemente, devela las asimetrías que atraviesan la representación, posicionando la práctica cartográfica desde otro lugar, desde donde valorar las voces de los agentes locales. La cartografía social (CS) se presenta como herramienta cualitativa capaz de representar significaciones del espacio en común, donde los conocimientos de los agentes locales y de los profesionales dialogan desde una perspectiva interdisciplinaria (Barragán-León, 2017). En nuestro caso, reconocemos un proceso de CS heterogéneo: no solo por la variedad de documentos cartográficos utilizados, que va desde los documentos catastrales, o mapas históricos, hasta documentos - la georreferenciación - elaborados por profesionales en diálogo con los vecinos; también, por los grados de apropiación y participación en el mismo mapeo. Precisamente, la convergencia entre enfoques y metodologías de CS con los sistemas de información geográfica participativa (SIGP) permite hoy generar representaciones del espacio particularmente útiles en los casos de deslinde (Mac Call, 2011); así, el SIGP propicia la interacción entre profesionistas y habitantes que, si bien no tienen conocimientos técnicos, son los mayores conocedores de su territorio, fomentando la visualización de nuevas configuraciones territoriales. La complejidad del conflicto en torno al parque ha implicado tratamientos específicos de la herramienta cartográfica, siempre orientados por las necesidades de los vecinos. De esta forma, enfatizando el intercambio de saberes, presenciamos un proceso donde SIGP y CS fortalecen la acción ciudadana y visión territorial.

\section{Estrategia metodológica}

Considerando nuestros intereses, así como el enfoque propuesto para abordar los efectos del conflicto, la estrategia metodológica se ha centrado en el significado y valores que subyacen a la acción de los agentes y, consecuentemente, en los efectos de la disputa, valorando el alcance del proceso de apropiación de la herramienta cartográfica. Abordamos el estudio desde un acercamiento cualitativo: recuperamos la postura de los agentes locales desde un enfoque de investigación-acción, recurriendo al diálogo entre lo que los agentes enuncian, y lo que hacen (Bourdieu y Vacquant, 2005). Para ello, la aplicación de entrevistas y revisión documental se complementa con observación participante, la cual se documenta en apuntes etnográficos, para luego ser sistematizada. Partiendo de la base de que no son los agentes quienes dan cuenta del sentido de una escena, sino su lectura relacional, hemos procurado mirarlos como sujetos ubicados en un entorno socioespacial e histórico (Giglia, 2012, p. 75). Se realizó la revisión documental, particularmente del material proporcionado por la Asamblea de Vecinos en Defensa del Parque Reforma Social (AVDPRS). Destaca la utilización de una encuesta (2013) ${ }^{4}$, además de documentos históricos, hemerográficos y cartográficos. La visión de los agentes ha sido profundizada gracias al análisis del discurso a partir de los documentos mencionados ${ }^{5}$ y de seis entrevistas semiestructuradas a miembros de la AVDPRS, las cuales tenían dos objetivos: esclarecer el sentido de la acción vecinal y reconocer el papel, conocimiento y apropiación de la herramienta cartográfica.

4 En la encuesta, aplicada a 144 usuarios por la AVDPRS en 2014 se pregunta: nombre y edad del encuestado, tiempo de ocupación en la vivienda, escrituras, motivo por el cual defiende el parque. Se agruparon las respuestas en tres categorías: A-Porque tiene un valor medioambiental en la ciudad y es importante para hacer ejercicio (73 reactivos). B-Porque es parte de nuestra historia (14). C-Porque es importante para la sociabilidad, el recreo y la comunidad (43). D-Porque es un espacio público (14).

5 Algunos documentos fueron recuperados del blog de la AVDPRS (Salvandoelparque: https://salvandoelparquerf.wordpress.com), mientras que otros se encuentran resguardados por la AVDPRS. 
La valoración del papel de la cartografía en la producción territorial sigue los pasos de la periodización del conflicto (Tabla 1) para comprender el vínculo entre este, el uso y apropiación de la cartografía y la producción territorial. Evidenciando los objetivos que subyacen a su utilización, proponemos una sistematización donde lo analizado en los testimonios nuevamente se contrasta con la observación participante de las actividades realizadas en 2019, entre las cuales destacamos: reuniones y actividades vecinales; un recorrido acompañado (Arias, 2017); los talleres de memoria y diagnóstico participativo organizados en colaboración con la Maestría en Planeación en Políticas Metropolitanas (MPPM) de la Universidad Autónoma Metropolitana-Azcapotzalco (UAM-A), en el marco del ejercicio de Planeación Aplicada ${ }^{6}$, Ecobarrio-Reforma Social 2030.

\section{El conflicto por la privatización del parque Reforma Social}

Reforma Social es una colonia popular autoconstruida por sus primeros habitantes en los años cincuenta del siglo pasado. Se localiza en la alcaldía Miguel Hidalgo, en el límite con el estado de México, cercana a Paseo de Reforma, una zona con gran capital económico y edificios corporativos de nivel internacional. A inicios del siglo XX, se estableció una mina de arena, cuyos alrededores fueron poblándose hasta que, en 1952, parte de los terrenos de la hacienda de los Morales fueron comprados por el Gobierno Federal. Junto con los habitantes que se asentaron en la colonia, había otros dos grupos de pobladores, un grupo que habitaba las cuevas de la mina, cuyos integrantes fallecieron en un derrumbe causado por las lluvias; y un tercer grupo, asentado en la barranca del río Tecamachalco, el cual fue desplazado hacia San Juan de Aragón a finales de los sesenta. La colonia surge sobre dos predios comprados por el Gobierno Federal: el primero, adquirido en 1945 al general Orozco Camacho (100.062,6 $\mathrm{m}^{2}$ ); el segundo corresponde a un terreno de la hacienda de los Morales, comprado en 1946 a Carlos Cuevas Lascurain (209.415,5 m²). En 1966 mediante decreto, se asignaron los lotes de la colonia Reforma Social.
En los terrenos de Cuevas Lascurrain, se trazaron las calles uno a la catorce de la colonia; mientras que en otro predio ubicado en el estado de México, se trazaron las calles de quince a veinte. El presidente Gustavo Díaz Ordaz (1966) oficializó el asentamiento desincorporándolo del terreno federal por medio de un decreto en pro de quienes lo habitaban (Navarro Jiménez, 2015) y, sobre el terreno obtenido rellenando la barranca del río Tecamachalco, surgió el parque (194,682 m²). En 1977, la sucesión de Cuevas Lascurrain (desde ahora, la Sucesión), interpuso un amparo frente al Gobierno Federal reclamando el derecho de propiedad sobre el predio en cuestión. Pero no fue hasta 2010, que el delegado Demetrio Sodi anunció la necesidad de acatar la sentencia del juicio de amparo y entregar el parque a la parte quejosa, que los vecinos conocieron el proyecto inmobiliario en torno al parque y emprendieron su proceso de organización.

\section{La activación del conflicto para la defensa del parque}

Cuando los vecinos supieron de la resolución del juicio de amparo - que reconoce la propiedad a la parte quejosa - se organizaron y, en junio de 2010, formaron la Asamblea de Vecinos en Defensa del Parque Reforma Social (AVDPRS), principal espacio de organización vecinal. Se instaló un primer campamento provisional. Entre las comisiones, la Jurídica tuvo un papel central, porque para revertir la sentencia había que demostrar que el río Tecamachalco - de propiedad federal y que fuera la referencia para el deslinde original del predio- corre entubado bajo el parque, lo cual confirmaba su carácter público. La AVDPRS necesitó reconstruir la historia del asentamiento para demostrar que el deslinde original fue correcto, lo cual pudo lograr gracias a la revisión histórica de mapas, documentos y a la memoria de los fundadores. Desde ese momento, la reconstrucción histórica de la colonia, la cartografía y el proceso jurídico, quedaron imbricados. El conflicto evidencia un mecanismo de identificación entre colonia y parque como espacio público y lugar de encuentro vecinal, que refleja los esfuerzos hechos para vivir en un entorno digno.

6 Para más información ver: http://mppm.azc.uam.mx/index.php/alumnos/ejercicios-de-planeacion-aplicada 
Figura 2

Domingo en el parque; al fondo edificios corporativos

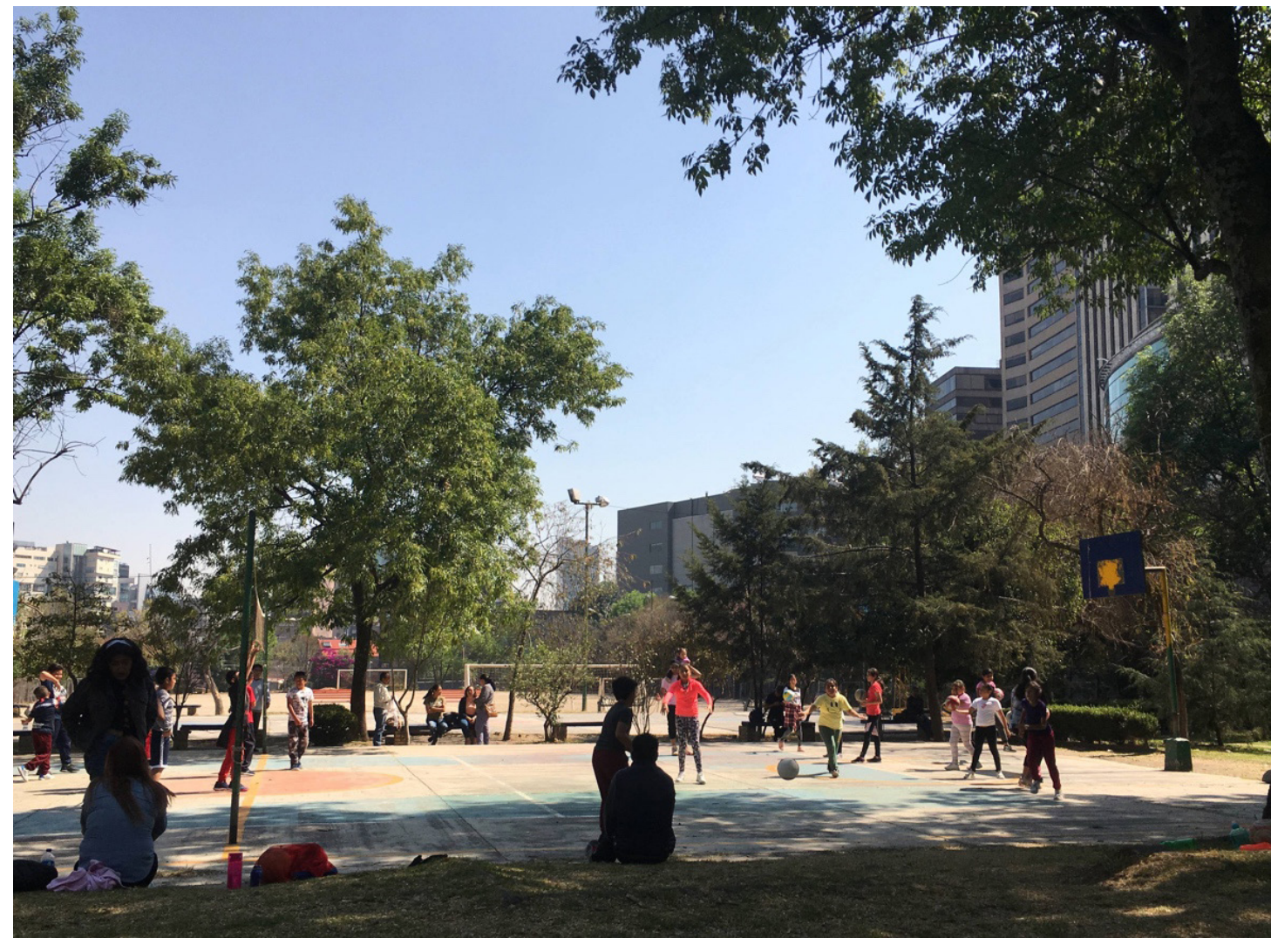

es la parte más bonita, porque aquí es como el centro de reunión y también de unión de todos [...] el verde te hace sentir muchas cosas y te despierta muchas otras, entonces para mí es importante porque esto le da vida a la colonia y por eso es tan importante conservarlo; si se va esto, [...] obviamente lo que sigue somos nosotros, y esa colonia se construyó con muchos esfuerzos [...] independientemente de que un área verde debe ser sagrada, es porque este es el espacio de la colonia: [...] a lo mejor en la casa no tenemos el espacio [...] o no tenemos agua, pero podemos venir a ver a los niños jugar... (comunicación personal con Araceli, enero 2020).

Una encuesta aplicada por la AVDPRS en 2014 confirma cómo el parque representa para los habitantes el lugar de la sociabilidad, memoria e identidad, y por ello tiene un valor universal, en tanto el cuidado del medioambiente es visto como bien común por parte de la ciudadanía. Por el contrario, para la Sucesión este tiene solo un valor estrictamente instrumental y se presenta como una excelente oportunidad de ganancia.

\section{Los actores involucrados}

En la disputa destacamos tres grupos de agentes: los vecinos de la AVDPRS, que logran vincularse con otros grupos ciudadanos, y deciden apostar por la figura del Comité Ciudadano ${ }^{7}$ y por el uso del Presupuesto Participativo para promover un proceso de apropiación por parte de los habitantes.

Los contrincantes de la Asamblea son representados por la Sucesión, que el fallo del juicio 577/77 reconoció como propietaria. La Sucesión se asoció con Inmobiliaria Leo para gestionar los permisos necesarios para realizar torres residenciales con capacidad para 1.200 departamentos.

Cuando el Gobierno Federal pierde el juicio de amparo $577 / 77$ y, por tanto, la propiedad del parque, los vecinos

7 El Comité Ciudadano, hasta 2020, fungía como el órgano de representación ciudadana de cada colonia en la Ciudad de México. Ver más información en https://www.iecm.mx/participacionciudadana/comites-ciudadanos/. 
Figura 3

El plano de INDAABIN refrenda la versión vecinal

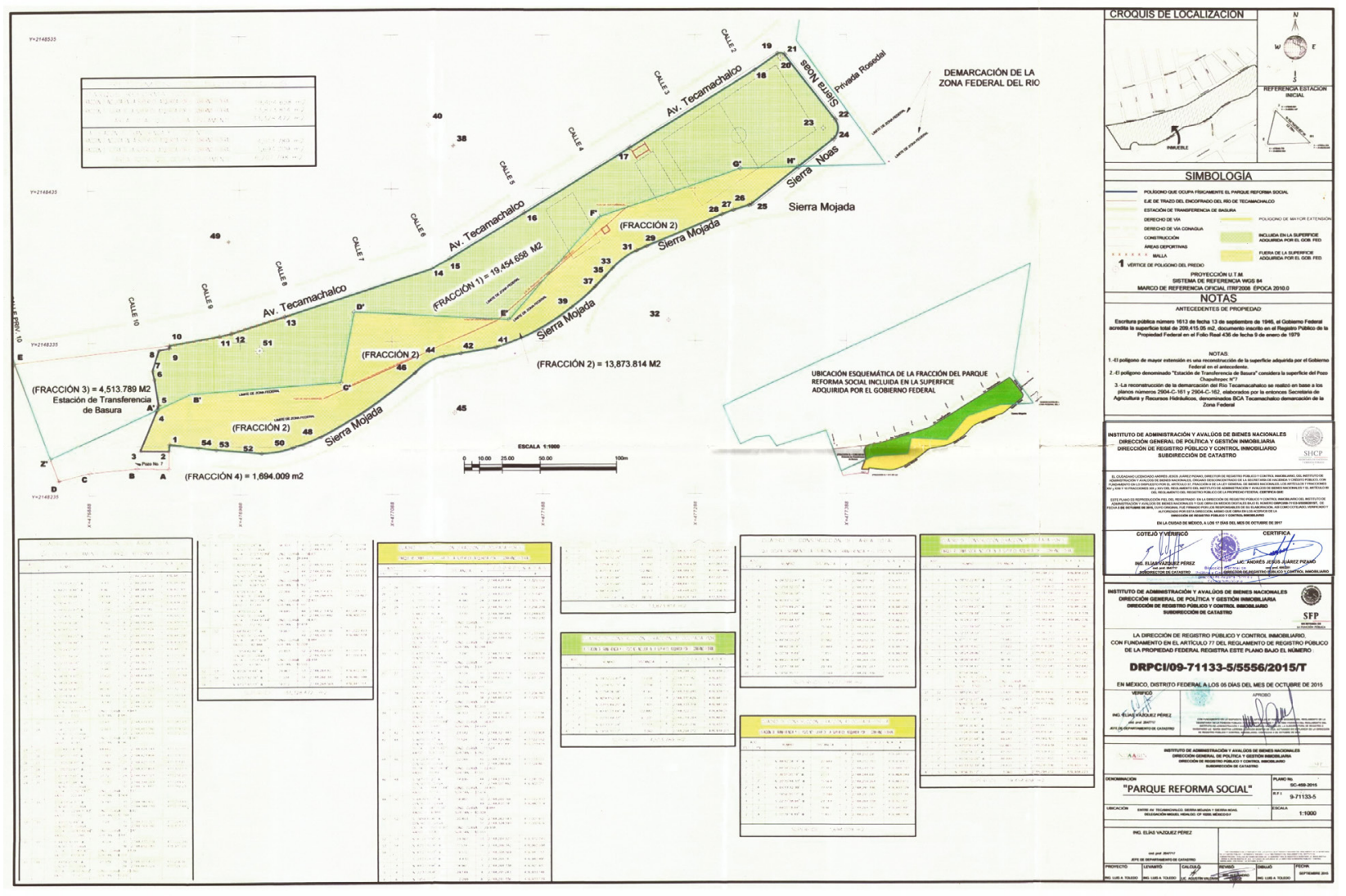

Nota. El plano fue elaborado por el INDAABIN a partir de las mesas de negociación. Fuente: Gentileza de la AVPDRS

asumieron su defensa como bien público. Sin embargo, aunque su incidencia en el conflicto parecía limitada, el Gobierno Federal tenía la potestad de determinar la propiedad del predio. Así, el Instituto de Administración y Avalúos de Bienes Nacionales (INDAABIN) coordinó una mesa de negociación y, en 2015, publicó un plano del parque que refrendaba la versión vecinal (Figura 3). A su vez, la Comisión Nacional de Agua (Conagua) fue la instancia responsable de reconocer el trayecto del río Tecamachalco y atestiguar el lindero del predio.

A nivel local, el gobierno de la ciudad y la alcaldía son las instituciones encargadas de generar e implementar los instrumentos de planeación y ordenamiento territorial, asentando el uso de suelo en el Programa Delegacional de Desarrollo Urbano (PDDUD-MH) y en el Programa Parcial de Desarrollo Urbano de Lomas de Chapultepec (PPDU-Lomas de Chapultepec).

\section{Estrategias y discursos}

En 2010, algunos miembros de la AVDPRS lograron ganar las elecciones del Comité Ciudadano. Por su parte, la Sucesión negoció con el Gobierno de la ciudad un convenio donde se prevé la cesión del 30\% de la superficie a cambio de la modificación de uso de suelo y del permiso para construir.

La Sucesión marcó entonces los hitos de la disputa: la definición de la propiedad del predio y el uso de suelo. La Asamblea implementó sus estrategias alrededor de estos ejes, integrando una tercera ruta y, en lugar de reaccionar a los embates de la Sucesión, apuntó a fomentar la participación y la apropiación del territorio por parte de los habitantes.

Mientras las posiciones de los dos grupos resultaban contrapuestas; la institución pública sostuvo una postura ambivalente: el Gobierno Federal no reclama la propiedad del predio, mientras los vecinos piden que la federación 
Tabla 1

Secuencia de las querellas jurídicas en torno al parque Reforma Social

\begin{tabular}{|c|c|}
\hline AÑo & EVENTO \\
\hline 1966 & El 10/06/66: Decreto por el cual se asignan a los colonos los lotes conformantes la colonia. \\
\hline 1977 & $\begin{array}{l}\text { Decreto que destina } 29.120 \mathrm{~m} 2 \text { para servicios públicos, deportivos y áreas arboladas al servicio de la ciudadanía y vecinos de la colonia. Se crea el } \\
\text { parque; la Sucesión interpone amparo. }\end{array}$ \\
\hline 1996 & $\begin{array}{l}\text { Sentencia del Tribunal Colegiado: el parque es desincorporado de los bienes de dominio público de la Federación para entregarse a la nueva } \\
\text { propiedad. Recursos por inejecución de sentencia, hasta } 2009 \text { cuando el gobierno realiza la entrega "oficial" del predio. }\end{array}$ \\
\hline 2002 & El 3 de junio el presidente Vicente Fox promulga el decreto que dejará sin efecto el decreto de 1977. \\
\hline 2013 & $\begin{array}{l}\text { La sentencia de amparo (532/2013) por la falta de asignación de uso de suelo del predio reconoce el uso de área verde previsto en los } \\
\text { instrumentos de planeación. }\end{array}$ \\
\hline 2013 & $\begin{array}{l}\text { La inmobiliaria "Viviendas Leo S.A. de C.V." (GRUPO HIR) interpone otro amparo (532/2013), por falta de asignación de uso de suelo del predio. El } \\
\text { Comité Ciudadano logra ser reconocido como tercer interesado. }\end{array}$ \\
\hline 2020 & En junio el amparo es rechazado. \\
\hline
\end{tabular}

actúe como árbitro y garante del bien común. El convenio entre el Gobierno de la ciudad y la Sucesión tendría que ser refrendado por la Asamblea Legislativa, pero, ya sea por la exposición pública del conflicto, o por la renovación del gobierno, la postura institucional cambió y en los instrumentos de planeación, como el Programa Delegacional de Desarrollo Urbano-Miguel Hidalgo (SEDUVI, 2008) y el Programa Parcial de Desarrollo Urbano-Lomas de Chapultepec (SEDUVI, 1992) el predio en cuestión continúa apareciendo como "Área Verde", con lo que se cancela el desarrollo inmobiliario.

En 2013, la defensa del parque cobró un significado radical para los vecinos. El evento definitorio fue el intento de bardear el parque por parte de miembros de la organización "Antorcha Popular" (hecho ocurrido el 23 de septiembre de 2013). Cuando los vecinos se enteraron de esta intención, no solo los activistas salieron a defender el predio, sino la colonia entera. La Asamblea no hubiera podido, por sí misma, detener la invasión, pero la defensa del parque develó un proceso de identificación.

cuando la gente tuvo la conciencia [...] es cuando hubo la invasión, ahí fue muy importante porque nunca nos habíamos planteado que tal vez pudieran venir a despojarnos... ¡nunca! La gente siempre decía [...] "es nuestro y no lo vamos a permitir", “pasarán por nuestros cadáveres” y esas cosas. Por eso fue tan extraordinario el movimiento: porque el día que se atrevieron, la gente se organizó a su modo [...] tocaron las campanas de la Iglesia, y empezaron a recorrer calles, chavos con la bicicleta, otros con sus coches gritando: ¡se están llevando el parque! Y la gente al unísono corrió al parque. La gente vino sin que la obligaran, vino porque llegaban los abuelitos en silla de ruedas, con andadera y con bastón, los chivatos con su palo [...] es muy bonita la historia (comunicación personal con Araceli, enero 2020).

Los vecinos reinstalaron el campamento y empezó un período de recrudecimiento de la disputa. Esta etapa se corresponde con el desplazamiento del Gobierno Local, cuyos representantes modificaron el discurso de la administración anterior. El gobierno delegacional de Víctor Romo se pronunció a favor de una expropiación con una declaración de utilidad pública: se habló de recuperar el espacio público; INDAABIN instituyó mesas de trabajo entre autoridades y colonos que concluyeron que la Sucesión, sin poder demostrar la posesión del parque, no podía modificar su uso de suelo. En estas mesas se reconocieron tres rutas: la expropiación, la declaración de juicio nulo o, una nueva compraventa.

La alcaldía resulta determinante para modificar la relación de poder a favor de los habitantes. Tal es el caso que, en 2018, la demarcación intentó dar mantenimiento al parque; la Sucesión, en franca contraposición, envió a la policía a detener a los trabajadores y al mismo alcalde Romo (Buscan darle uso habitacional a parque Reforma Social: Romo, 2018). 
Figura 4

Taller de memoria e identidad: realizado con habitantes y vecinos desplazados de la Barranca

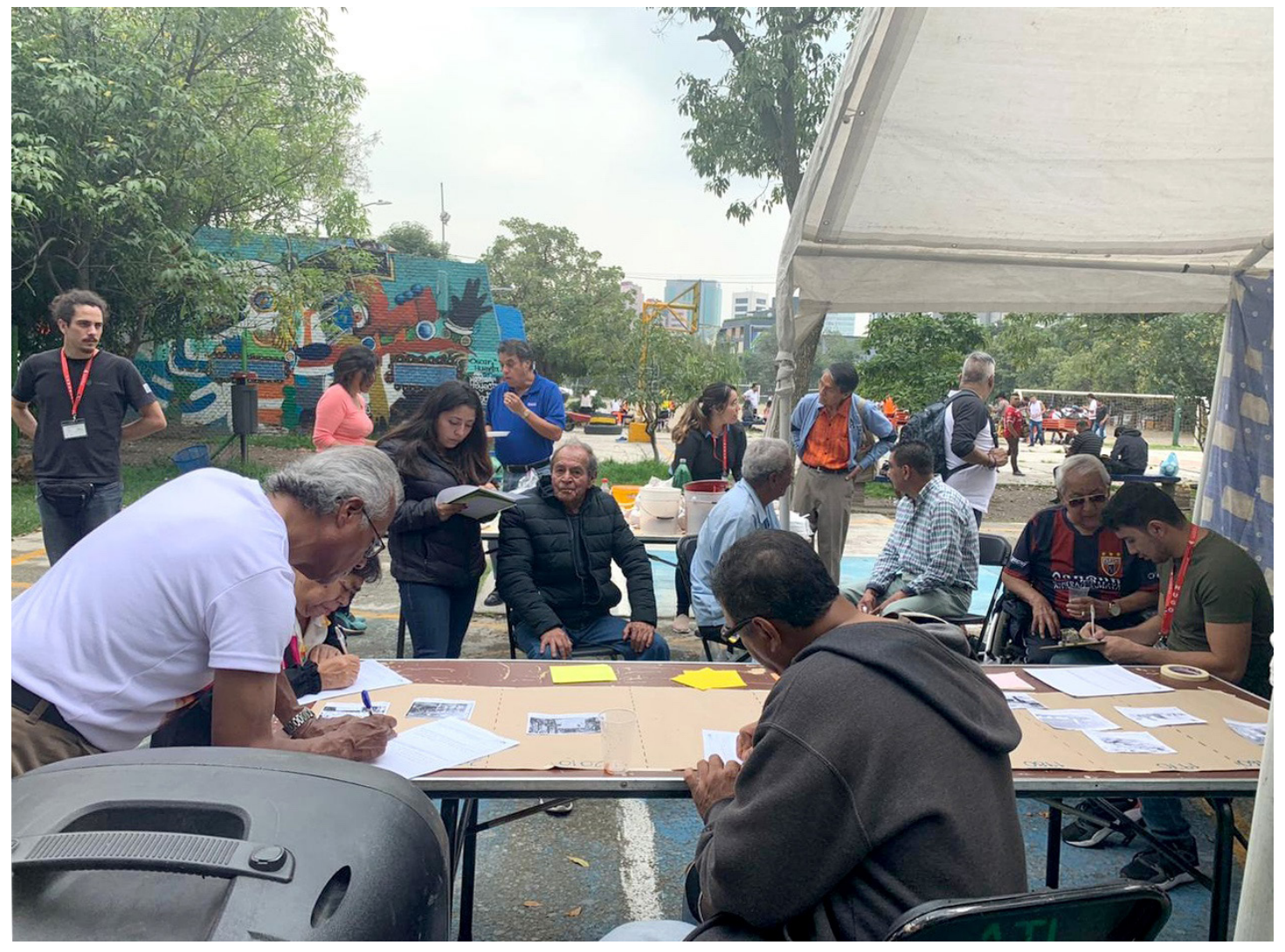

Finalmente, aunque el gobierno local no pudo resolver la situación jurídica del predio, intervino en la asignación de uso de suelo y fue el actor que medió entre los vecinos y el nivel federal.

El proceso de apropiación se vio reforzado y este evento marcó el pasaje desde la defensa del parque al territorio. En la última etapa se fomentó la visualización de proyectos futuros que el Comité Ciudadano implementaría con otros agentes, en particular, con la MPPM para la elaboración de un programa piloto.

\section{Repertorio de acción}

El repertorio de acciones implementadas por la AVDPRS es amplio. La Asamblea estrechó vínculos con otras organizaciones y de esta sinergia surgió la propuesta de presentarse a las elecciones de Comités Ciudadanos. Para oponerse a la instalación del gas FENOSA, los vecinos se articularon con grupos ciudadanos de la Condesa. También se implementó una estrategia de comunicación en las redes sociales y se abrió un blog. Se organizaron campañas de información y la Comisión Jurídica preparó el material para armar una demanda legal. Se realizaron marchas y mitines a los Tribunales involucrados en el juicio, se produjeron videos para difusión en los medios televisivos. Los miembros de la Asamblea buscaron profesionales que los orientasen, sobre todo en lo referido al proceso judicial. Se procuró tejer alianzas con otros procesos de defensa territorial: el caso del parque se expuso en el Tribunal Permanente de los Pueblos (TPP) (http://permanentpeoplestribunal.org/?lang=es); los vecinos participaron en eventos con otros grupos, construyendo una lectura de los procesos urbanos donde, el del parque resultó un caso entre muchos de despojo y corrupción. Se tuvieron mesas de negociación con el Gobierno Local e INDAABIN. El tránsito de la defensa del parque a la colonia, con el apoyo de otros actores, marcó el desplazamiento desde la contraposición a la visualización de propuestas para el Gobierno Local.

\section{Cartografía y conflicto: entre apropiación y proyecto}

Los vecinos se apropiaron del tema cartográfico para reforzar los argumentos a favor del carácter público del predio. 


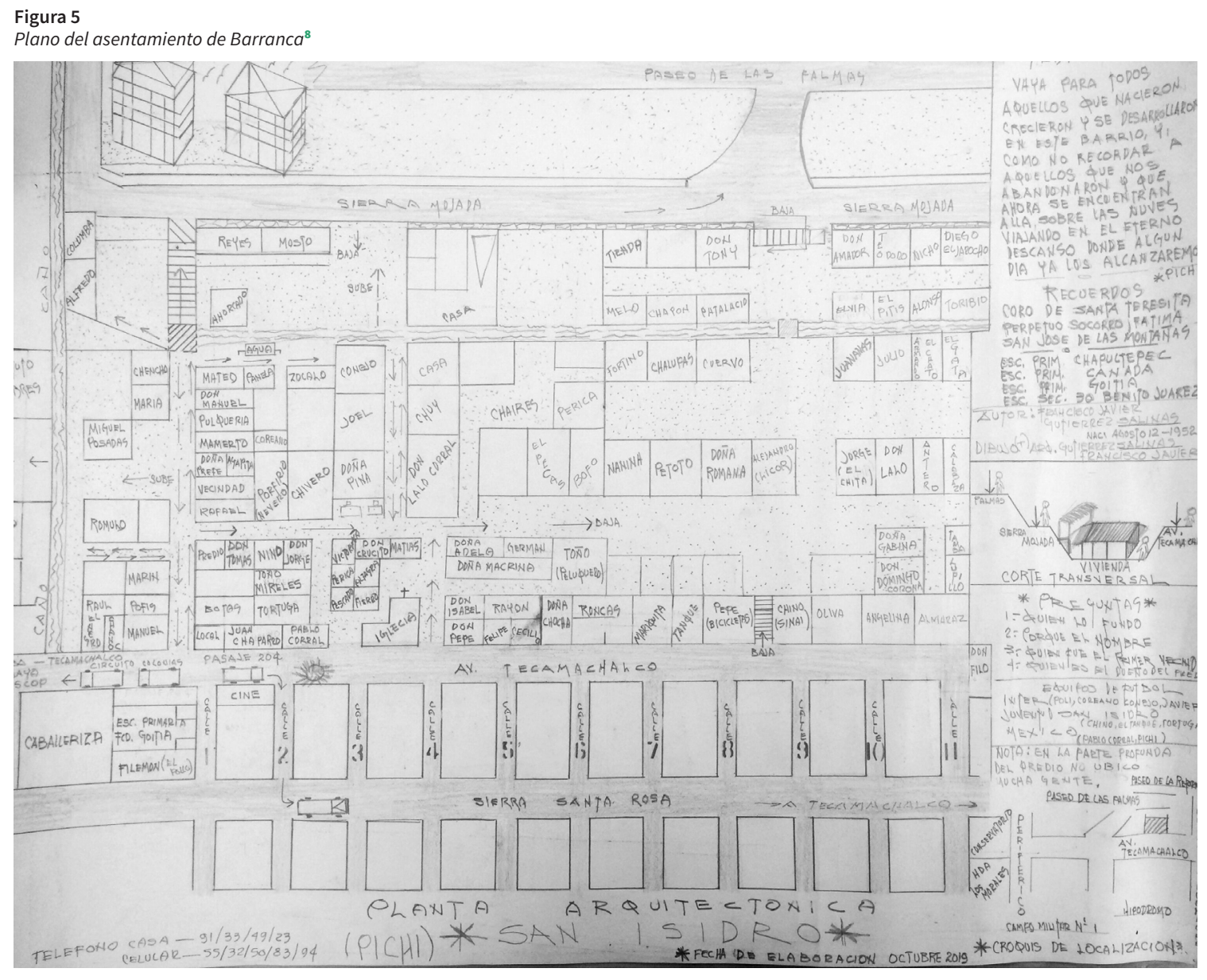

Nota. El plano fue elaborado por el INDAABIN a partir de las mesas de negociación. Fuente: Gentileza de la AVPDRS

En este sentido, es posible reconocer tres etapas del conflicto donde la utilización del material cartográfico apuntaló las estrategias para defender el parque: deslinde y demostración de la propiedad pública del predio; definición del uso de suelo como área verde; y la proyección territorial (Tabla 2).

Los primeros mapas proporcionaron argumentos para el litigio legal: en los mapas históricos de la hacienda de los Morales estaban los elementos para reconstruir la circulación del río hoy entubado y, con base en este, la localización del parque. Los mapas catastrales, sin referencias territoriales, fueron interrogados para comprobar localización y superficie total. En estos primeros ensayos, los vecinos necesitaban apoyo para leer los mapas; además, tenían que acreditar la precisión de su reconstrucción histórica. Con los datos recabados, complementados con el material cartográfico obtenido en el Archivo Agrario de la Nación (AAN) y en el Archivo Histórico y Biblioteca Central del Agua de Conagua, se reconstruyó el cauce del río. Se puede observar en ello un proceso participativo coordinado por la Asamblea con algunos vecinos profesionales que, aunque no corresponde a un producto cartográfico específico, sienta las bases del proceso de apropiación.

Sí, la gente conoce esos mapas, nosotros se los hemos puesto en las asambleas y les hemos dicho dónde está el río, los quiebres famosos, donde están los respiradores, algunos se han interesado y hemos ido a hacer recorridos. [...] eso enamoró un poco a mucha gente (comunicación personal con Araceli, enero 2020).

Sucesivamente, el uso de la cartografía fue cada vez más específico y concierne a la instrumentación de la demanda

8 Este plano se presentó en el taller de identidad y memoria y muestra al asentamiento en la Barranca, identificando cada casa con un apodo. 
Tabla 2

Planos y mapas que figuran a lo largo del conflicto

\begin{tabular}{|c|c|c|c|}
\hline Mapas/planos & Utilizado en juicio & Etapa-argumento que refuerza & $\begin{array}{l}\text { Memoria y } \\
\text { apropiación }\end{array}$ \\
\hline $\begin{array}{l}\text { Escritura cancelada por el juicio 577/77. } \\
\text { Fecha: febrero } 1977 \\
\text { Autor: SAHOP, Dirección de Catastro de } \\
\text { Propiedad } \\
\text { Escala 1:2.000 }\end{array}$ & Plano cancelado por el juicio & ETAPA I. Propiedad & $x$ \\
\hline $\begin{array}{l}\text { Sin Título. } \\
\text { Fecha: } 1950 \\
\text { Autor: Secretaría de Recursos Hidráulicos } \\
\text { Sin escala }\end{array}$ & Sí & $\begin{array}{l}\text { ETAPA I. Propiedad } \\
\text { Existencia y ubicación del río Tecamachalco }\end{array}$ & $x$ \\
\hline $\begin{array}{l}\text { Programa Delegacional de Desarrollo Urbano } \\
2009 . \\
\text { Autor: Delegación M. Hidalgo/SEDUVI } \\
\text { Escala: 1:10.000 }\end{array}$ & Sí & ETAPA II. Uso de suelo: Área Verde & $X$ \\
\hline $\begin{array}{l}\text { Programa Parcial de Desarrollo Urbano-Lomas } \\
\text { de Chapultepec } 1992 \\
\text { Autor: Delegación M. Hidalgo/SEDUVI } \\
\text { Escala: 1:10.000 }\end{array}$ & Sí & ETAPA II. Uso de suelo: Área Verde & \\
\hline $\begin{array}{l}\text { Plano del Parque Reforma Social, } 2005 \\
\text { Autor: INDAABIN } \\
\text { Escala: 1:2.000 }\end{array}$ & & ETAPA II. Confirma la versión de la AVDPRS & \\
\hline $\begin{array}{l}\text { Plano-croqui del Asentamiento en la Barranca } \\
\text { del Río Tecamachalco. } \\
\text { Fecha: noviembre } 2019 \\
\text { Autor: Arq. Gutiérrez y desplazados } \\
\text { Sin escala (Fig.3) }\end{array}$ & & $\begin{array}{l}\text { ETAPA III. Propiedad. Existencia del Río. } \\
\text { Argumento: la Sucesión no posesión del predio. } \\
\text { Reconstrucción de la memoria }\end{array}$ & $\begin{array}{l}\text { Elaborado a mano } \\
\text { por los vecinos } \\
\text { desplazados en } \\
1966 .\end{array}$ \\
\hline $\begin{array}{l}\text { Defensa del Parque Reforma Social. Plano } \\
\text { Comparativo } \\
\text { Fecha: } 13 / 09 / 2015 \\
\text { Autor: Geocomunes } \\
\text { Geoportal de Geocomunes. Mapa Dinámico y } \\
\text { poster (Figura 2) }\end{array}$ & & $\begin{array}{l}\text { ETAPA III. Propiedad. Ubicación del predio. Geo- } \\
\text { referenciación del predio con base en fuentes } \\
\text { históricas y catastrales }\end{array}$ & $x$ \\
\hline
\end{tabular}

por fraude procesal que, aunque no procede, permite contrastar los pasajes del juicio de amparo 577/77 con los argumentos de la contraparte. Se utilizó el SIG para georreferenciar los elementos del litigio legal: la ubicación del antiguo cauce del río Tecamachalco, lindero de referencia en el litigio, el cálculo de las superficies mencionadas en la compraventa, su uso en el juicio 577/77. Se definió de manera conjunta, cuál sería la representación cartográfica óptima para contrastar la postura de la Sucesión (se optará por una presentación PPT, combinando deslinde y argumentación). Se instalaron mesas de negociación con INDAABIN y los vecinos acompañaron al personal del instituto en los recorridos para el levantamiento del predio.

ellos mismos emitieron el plano, han de estar arrepentidos [...] después fue otro vecino [...] y le dijeron que ya iba a ser diferente porque había sido modificado. [...] no les quedó de otra, porque ese vecino no se dejó y les expuso: ¿no? Entonces me voy a Asuntos Legales, porque ese plano, yo lo quiero porque ya lo tiene otro vecino, y certificado: así lo quiero yo, y entonces tenemos dos (comunicación personal con Gustavo, octubre 2019).

En esta etapa, la georreferenciación del lindero fue realizada por un geógrafo, habitante de la comunidad, quien se integrará al núcleo fundador del colectivo Geocomunes (Figura 5).

Mapas y planos, que proporcionan la información sobre uso de suelo, son productos técnicos que tampoco contemplan la visión de los vecinos, pero su utilización profundiza el proceso de acercamiento a esta herramienta inaugurado en la primera etapa.

9 La georreferenciación del parque consta de una presentación Power Point que se utilizaba en el marco del juicio y fue realizada por un geógrafo, quien fue miembro fundador del colectivo Geocomunes. 
Figura 5

Mapa: Conflicto parque Reforma Social

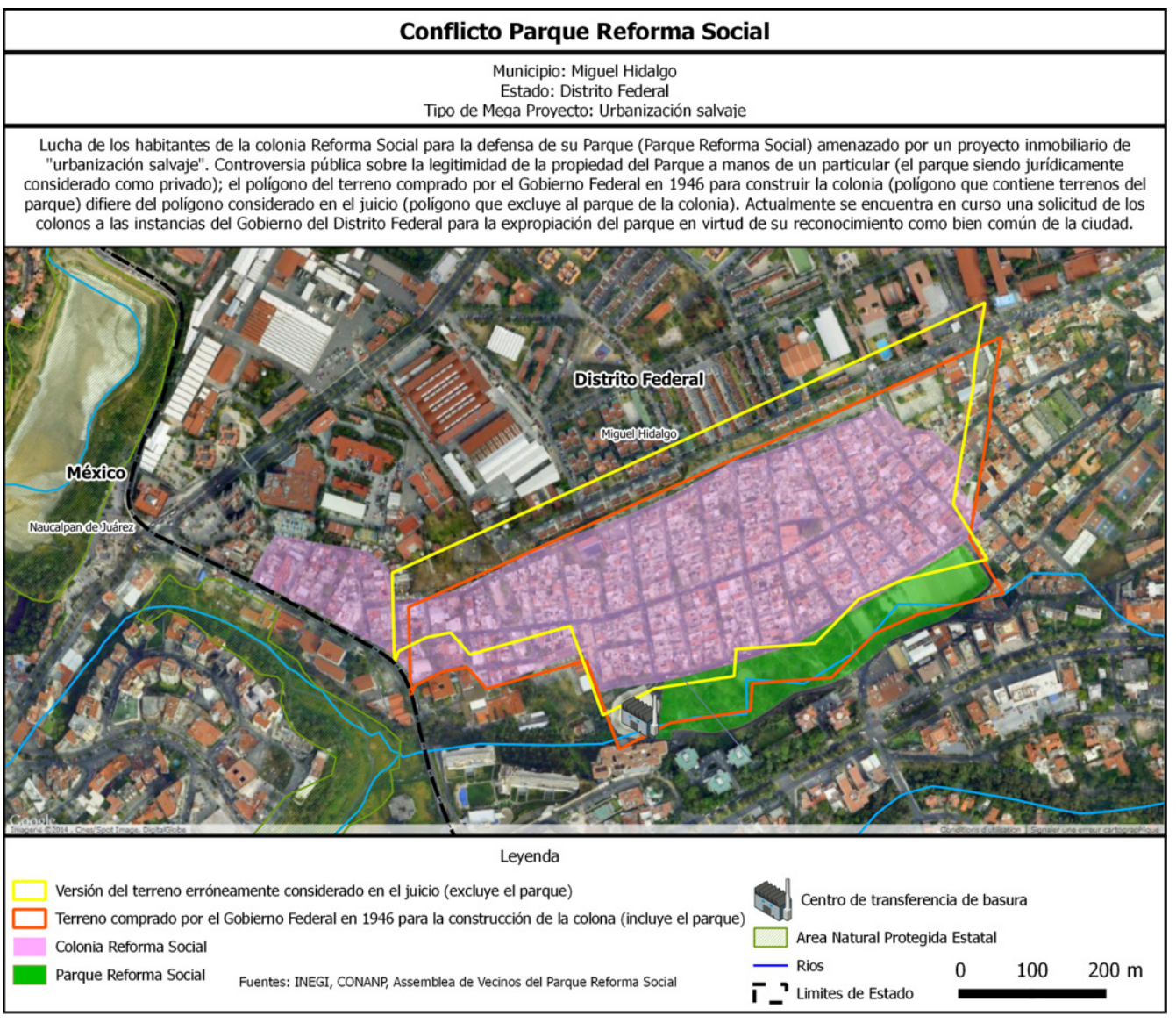

Nota. El mapa fue realizado para la sesión del TPP que tuvo lugar en el parque.

Fuente: Geoportal de Geocomunes (http://geocomunes.org)

Finalmente, el Comité Ciudadano fomentó la apropiación territorial y, el tema cartográfico se afianzó en un proceso participativo novedoso. Se realizaron actividades culturales y se avanzó con la elaboración de un diagnóstico participativo de la colonia realizado con la Universidad Pública (UAM-A). Entre los talleres realizados para ello, cabe mencionar el de "Identidad y memoria" (Figura 3), en el cual, además de reconstruir el paisaje y los eventos, los vecinos desplazados expusieron un plano realizado a mano que representaba el asentamiento instalado en la Barranca (Figura 4). El Comité Ciudadano, además de utilizar el presupuesto participativo para contratar una barra de abogados que atendiera el desenlace jurídico de la querella, impulsó el diagnóstico participativo de las problemáticas de la colonia, como primer paso para llegar a gestionar con las autoridades competentes los proyectos propuestos por los habitantes.

\section{Conclusiones. El parque, la cartografía, el conflicto y su productividad}

En el artículo hemos analizado la disputa por la privatización del parque Reforma Social, y nos hemos centrado en los efectos de la productividad territorial, así como en el papel de la cartografía en la controversia. Los habitantes de Reforma Social no son actores homogéneos, si bien la AVDPRS es la agrupación con mayor apoyo, la posibilidad de defender la condición pública del predio se debe a la participación del conjunto de vecinos. Como en una figura metonímica, para los vecinos el parque es a la colonia, como la parte es al todo. Recientemente, el Comité Ciudadano implementó una estrategia para conseguir un doble objetivo: mientras mantienen comunicación con el Gobierno Local, conociendo su funcionamiento administrativo desde una nueva perspectiva; gracias al Presupuesto Participativo propusieron la definición de una estrategia jurídica para 
asentar la propiedad del parque. En este sentido, también la colaboración con la UAM-A reforzó la capacidad de los vecinos de decidir acerca de su propio territorio.

El mayor efecto fue el incremento de las interacciones entre los vecinos, con procesos de cohesión y apropiación del territorio, que abonan más allá del arraigo a las perspectivas a futuro. Su expresión espontánea es apreciable cuando Antorcha Campesina intentó invadir el parque y los vecinos salieron a repeler la acción. Ninguna agrupación, por sí misma, hubiera podido detener la invasión: solo la participación de los vecinos garantizó la defensa del carácter público del parque. El evento evidencia algo que ya estaba presente, sentando un proceso participativo de mayor alcance, poniendo de manifiesto el sentimiento de apego, la percepción del despojo y la injusticia. Logró cohesionar a los habitantes y cristalizar el hecho de que el parque es importante para todos. Así mismo, los militantes aprendieron a reconocer las distintas modalidades de participación y la posibilidad de movilizarlas: se volvieron expertos - autodidactas - de los procedimientos administrativos y normativos, experimentando una forma híbrida de vinculación entre lo gubernamental y lo ciudadano. Algunos miembros de la AVDPRS se movieron desde la postura meramente activista, a ocupar la figura del Comité Ciudadano u otros espacios en la administración local, para promover acciones de cuidado del ambiente. En cuanto a la norma jurídica, los vecinos tuvieron que confrontarse con este aspecto por la genealogía misma del conflicto, sin embargo, consideran que la solución es política: más que confianza en el sistema de justicia, son conscientes de que el conocimiento del sistema jurídico representa una herramienta para lograr sus objetivos.

La CS respalda el proceso organizativo. Distinguimos un proceso de reconstrucción de la historia de la colonia donde, más que la elaboración de un producto, observamos una construcción colectiva de la memoria, complementada por la información reportada en los mapas históricos, que comprueba la propiedad pública del predio. En la segunda etapa se utilizaron mapas técnicos y gubernamentales. Los vecinos usaron los mapas a su favor, comprendiendo el papel de la cartografía en los instrumentos de ordenamiento territorial y más allá del foco en el parque, gradualmente pasaron de su defensa, a visualizar la colonia entera. Recientemente, la cartografía es parte de un proceso participativo, ahora volcado al proyecto, desde el mapa catastral, documento de propiedad ajeno al territorio, los vecinos proyectan ahora su territorio.

En la controversia, el parque es lo que está en disputa y el teatro del conflicto: punto de encuentro entre vecinos e invasores. En 2013, la Sucesión envió a la policía a detener al mismo delegado, porque, al dar mantenimiento al parque, el personal delegacional violaba una propiedad privada. Estos eventos evidencian los intentos de la contraparte por ejercer un control material del predio, y la concurrencia de las territorialidades. La memoria complementa y refuerza la defensa física del territorio por la vía simbólica, y la pertenencia se expresa en la cohesión, en el reconocimiento del valor ambiental, así como en el cuidado que los vecinos han dedicado al parque.

Finalmente, observamos un proceso de identificación con el futuro del parque, por lo cual, desde nuestra perspectiva, el producto más importante del conflicto es el paso de la reacción frente a un evento considerado injusto, a la construcción de un proceso comunitario que amplía la mirada hasta abarcar el futuro del territorio.

\section{Referencias bibliográficas}

Aliste, E. (2010). Territorio y ciencias sociales: trayectorias espaciales y ambientales en debate. En E. Aliste y A. Urquiza (Eds.), Medio ambiente y sociedad. Conceptos, metodologías y experiencias desde las ciencias sociales y humanas (pp. 55-76). RIL editores. http://dx.doi. org/10.4067/S0718-34022010000300011

Arias D. (2017). Etnografía en movimiento para explorar trayectorias de niños y jóvenes en Barcelona. Revista de Antropología Social, 26(1). http://dx.doi.org/10.5209/ RASO.56044 
Azuela, A. (2014). Conflictos urbano-ambientales en América Latina. Revista América Latina en Movimiento, (497). https://www.nodal.am/2014/07/conflictos-urbanoambientales-en-america-latina-por-antonio-azuela/

Barragán-León A. N. (2019). Cartografía social: lenguaje creativo para la investigación cualitativa. Sociedad y economía, (36), 139-159. https://doi.org/10.25100/sye.v0i36.7457

Bredenoord, J., Lindert, P. V., \& Smets, P. (2014). Affordable Housing in the Urban Global South: Seeking Sustainable Solutions. Routledge. https://doi. org/10.4324/9781315849539

Bourdieu, P. y Wacquant, L. (2005). Invitación a una sociología reflexiva. Siglo XXI.

Buscan darle uso habitacional a Parque Reforma Social: Romo (17 de noviembre 2018). Excelsior. https://www. excelsior.com.mx/comunidad/buscan-darle-usohabitacional-a-parque-reforma-social-romo/1279011

Castells, M. (1974). Movimientos sociales urbanos. Siglo Veintiuno de España.

Connolly, P., (2009). ¿Los mapas son ciudades? La cartografía como prefiguración de lo urbano. En L. Martínez Carrizales y T. Quiroz Ávila (Coord.), El espacio, presencia y representación. Universidad Autónoma MetropolitanaAzcapotzalco Ed.

Delgado Peña, J. J., Campoy Gómez, R. y Subires Mancera, M.P. (2015). Geografía, TICs e inclusión social: empoderamiento ciudadano desde el ámbito educativo para una regeneración urbana. Cuadernos Geográficos 54(1), 307-336. https://dialnet.unirioja.es/ejemplar/403014

De Matteis, G. y Governa. F. (2005). Territorio y territorialidad en el desarrollo local. La contribución del modelo slot. Boletín de la A.G.E., (39), 31-58. https://bage.agegeografia.es/ojs/index.php/bage/article/view/498

Di Méo, G. (1998). Géographie sociale et territoires. Nathan Ed.
Di Méo, G. y Buléon, P. (Dir.) (2005). L'espace social. Lecture géographique des sociétés. Armand Colin.

Dos Santos, B. (1991). Una cartografía simbólica de las representaciones sociales. Prolegómenos a una concepción posmoderna del derecho. Nueva Sociedad (116),18-38. http://www.boaventuradesousasantos.pt/media/ pdfs/Cartografia_simbolica_NuevaSociedad.PDF

GeoComunes (2020). Cartografía colaborativa en defensa de los bienes comunes. Visualizador de la Zona Metropolitana de la Ciudad de México. http://132.248.14.102/maps/213/view

Giglia, A. (2012). El habitar y la cultura. Perspectivas teóricas y de investigación. Anthropos y UAM-Iztapalapa.

Gwynne, R.N. (1999). Globalization, Neoliberalism and Economic Changes in South America and Mexico. En R.N. Gwynne \& C. Kay (Ed.), Latin America Transformed: Globalization and Modernity (pp. 39-63). Arnold

Harley, D. (2005). La nueva naturaleza de los mapas. Fondo de Cultura Económica.

Harvey D. (2013). Ciudades rebeldes del derecho de la ciudad a la revolución urbana. Akcal.

Instituto Electoral de la Ciudad de México, IECM (20 de marzo de 2020). Participación ciudadana. https://www.iecm. $\mathrm{mx} /$ participacionciudadana/comites-ciudadanos

Instituto Nacional de Estadística y Geografía, INEGI (2010). Censo de población y vivienda 2010. https://www.inegi. org. $\mathrm{mx} /$ temas/mg/

Mac Call, M. K. (2011). Mapeando el territorio: paisaje local, conocimiento local, poder local. En G. Bocco, P.S. Urquijo y A. Vieyra (Eds.), Geografíay Ambienteen América Latina. UNAM.

Melé, P. (2003). Introduction: conflits, territoires et action publique. En P. Melé, C. Larrue y M. Rosemberg (Coords.), Conflits et territoires, tours, presses (pp. 1332). Universitaires François Rabelais. https://books. openedition.org/pufr/1827?lang=it 
Melé, P. (2008) Conflits et controverses: de nouvelles scènes de production territoriale? Isabelle Garat, Raymonde Séchet, Djémila Zeneidi. Espaces en (trans)action, PUR, p. 239-250. https://halshs.archives-ouvertes. fr/halshs-01423812/document

Melé, P. (2016). ¿Qué producen los conflictos urbanos? En F. Carrióny J. Erazo (Coord.), El derecho a la ciudad en América Latina, visiones desde la política México (pp. 127-158. UNAM, Coordinación de Humanidades, PUEC, CIALC, IDRC/CRDI.

Melucci A. (1999). Acción colectiva, vida cotidiana y democracia. El Colegio de México.

Merlinsky, G. (2013). La espiral del conflicto. Una propuesta metodológica para realizar estudios de caso en el análisis de conflictos ambientales". En G. Merlinsky (Comp.), Cartografías del conflicto ambiental en Argentina (pp. 61-82). Fundación CICCUS.

Mora-Páez, H., Jaramillo, E. (2004). Aproximación a la construcción de cartografía social a través de la geomática. Ventana Informática, (11). http://www.beu.extension. unicen.edu.ar/xmlui/bitstream/handle/123456789/348/ Mora-Paez\%2C\%20Jaramillo_CartografiaSocial\%20 $\mathrm{y} \% 20$ geomatica.pdf? sequence $=1 \&$ isAllowed $=\mathrm{y}$

Morales Guzmán, J. C. (2017). Disputas ciudadanas en torno a los megaproyectos urbanos en la Ciudad de México. Espacialidades. Revista de Temas Contemporáneos sobre lugares, Política y Cultura, 7(2), 35-62. https:// www.redalyc.org/pdf/4195/419553524002.pdf

Navarro Jiménez, F. J. (2013). Territorios autoproducidos frente al urbanismo neoliberal: el caso de la colonia Reforma Social en la Ciudad de México. [Tesis de grado de Licenciatura de Geografía Humana, UAM-Unidad Iztapalapa, México] http://dcsh.izt. uam.mx/licenciaturas/geografia_humana/wp-content/ uploads/2014/11/Tesina-Francisco-Navarro-2013.pdf
Osorno-Covarrubias J., Couturier S. y Ricárdez M. (2015). El rol de la geografía y sus hibridaciones recientes frente a la crisis de sustentabilidad global. Boletín de la Asociación de Geógrafos Españoles-Bage,(69),93-112.https://doi.org/10.21138/bage.1891

Piceno, M. (2013). Reconocimiento y defensa del espacio público frente a procesos de urbanización neoliberal: el caso del parque reforma social [Tesis para grado de Licenciatura en Geografía no publicada]. Universidad Nacional Autónoma de México, México.

Piceno, M., Couturier, S., y Ricárdez, M. (5-10 de mayo de 2014). El SIG participativo como técnica de defensa de los espacios públicos urbanos: la experiencia de la asamblea de vecinos en defensa del parque Reforma Social, Ciudad de México [Sesión de conferencia]. XIII Coloquio Internacional de Geocrítica "El control del espacio y los espacios de control”, Barcelona, España.

Raffestin C. (1999). Paysages construit et territorialités, Convegno internazionale Disegnare paesaggi costruiti. DIUPRA, Politecnico di Milano, Italia.

Secretaría de Desarrollo Urbano y Vivienda, SEDUVI (1992). Programa Parcial de Desarrollo Urbano Lomas de Chapultepec. http://www.data.seduvi.cdmx.gob. mx/portal/index.php/programas-de-desarrollo/ programas-parciales

Secretaría de Desarrollo Urbano y Vivienda, SEDUVI (2008). Programa Delegacional de Desarrollo Urbano Del. Miguel Hidalgo. http://www.data.seduvi.cdmx.gob.mx/portal/ index.php/programas-de-desarrollo/programasdelegacionales

Simmel, G. (2010). El conflicto. Sociología del antagonismo. Sequitur.

Zamorano Villareal, C. (2019). Movimientos sociales urbanos en la Ciudad de México en el Siglo XXI. ¿Activismo encauzado al derecho versus acción prefigurativa? Revista Desacatos, (61), https:// desacatos.ciesas.edu.mx/index.php/Desacatos/ article/view/2130 